\title{
Research
}

\author{
Abigail Sabey and Holly Hardy
}

\section{Views of newly-qualified GPs about their training and preparedness:}

\author{
lessons for extended generalist training
}

\begin{abstract}
\section{Background}

General practice is becoming increasingly

complex due to an ageing population with multiple morbidities and the shift of services from secondary to primary care, yet GP training remains largely the same. Extended training is now recommended, initially proposed as a fourth GP specialty trainee year, but more recently as a broad-based 4-year specialty training programme.
\end{abstract}

\section{Aim}

To explore the views of newly-qualified GPs about their training and preparedness for specific aspects of the GP's role.

\section{Design and setting}

Qualitative study with newly-qualified GPs who qualified with Severn Deanery between 2007 and 2010.

\section{Method}

Semi-structured interviews with $18 \mathrm{GPs}$ between November 2011 and April 2012.

\section{Results}

Gaining experience in a variety of primary care environments widens insight into patient populations as well as helping GPs develop adaptability and confidence, although this is not routinely part of GP training. However, alongside variety, having continuity with patients in practice remains important. Opportunities to be involved in the management of a practice or to take on substantial leadership roles also vary widely and this may limit preparedness and development of generalist skills.

\section{Conclusion}

Extended training could help prepare GPs for the current challenges of general practice. It could ensure all trainees are exposed to a greater variety of primary care settings including those outside GP practice, as well as experience of business, finance, and leadership roles. Collectively, these changes have the potential to produce GPs with both generalist and enhanced skills, who are better prepared to work collaboratively across the organisational boundaries between primary. secondary, and community care.

\section{Keywords}

family practice; general practice; leadership; primary health care; qualitative; training.

\section{INTRODUCTION}

The job of a GP is fundamentally different from what it was 10 years ago. In the past decade, demographic changes, patient and societal expectations, medical advances, and new technologies have all contributed to the transformation of health care, driving changes in health policy and healthcare systems towards expansion of communitybased services as a means to improve health, raise quality, and reduce costs of care. $^{1-3}$ Where once GPs expected to work mainly in a single practice, which offered stability and continuity of colleagues and patients, newly-qualified GPs may now work in a variety of settings that deliver primary care closer to patients. Furthermore, as well as looking after patients' health, GPs must be competent in leadership and management of the organisations in which they work and are now involved in commissioning health services for their populations (Health and Social Care Act 2012), further transforming the nature of GP work.

Aside from new national curriculum and assessment processes for $\mathrm{GPs}^{4}{ }^{4}$ as part of reform across postgraduate specialist training, ${ }^{5}$ GP training has remained largely the same with trainees completing a variety of hospital posts and an apprenticeship period in general practice. Length of training has also remained the same despite an expanding curriculum and the ever more demanding and complex nature of the job.
The Shape of Training review ${ }^{6}$ proposed to overhaul the structure of all postgraduate medical training to ensure the medical workforce can meet the demands of a future NHS with fewer boundaries between sectors, bringing a focus on capabilities rather than competencies. This included the proposal for all specialist training to be for a minimum of 4 years, which is in line with the 2012 RCGP recommendation to extend GP training with at least 24 months spent in primary care. ${ }^{7}$ This was accepted by Medical Education England, although funding for this remains uncertain.

This study sought to explore the views of newly-qualified GPs about their training and experiences as newly-qualified GPs. The data were collected as part of a wider study about preparedness for practice and commissioning. The findings of this study may offer timely insight for those responsible for planning extended training.

\section{METHOD}

This article reports findings from a larger study; the method has been reported elsewhere. $^{8}$ In summary, the aim of this qualitative study was to explore personal experiences and views about GP training. Individual semi-structured interviews were chosen over focus groups to maximise the depth of discussion with each GP and to capture the individual narrative of their early years in general practice. Telephone interviews were offered based on previous
A Sabey, BSc, MSc, PGCE, senior lecturer, Faculty of Health and Applied Sciences, University of the West of England, Bristol. H Hardy, FRCGP. GP and associate postgraduate dean, Severn Postgraduate Medical Education, Health Education South West, Bristol.

\section{Address for correspondence}

Abigail Sabey, University of the West of England, Faculty of Health and Applied Sciences, Alexandra
Warehouse, Gloucester GL1 2LG, UK.

E-mail: abby.sabeyduwe.ac.uk

Submitted: 6 May 2014; Editor's response: 9 June 2014; final acceptance: 29 July 2014 CBritish Journal of General Practice This is the full-length article (published online 30 Mar 2015) of an abridged version published in print. Cite this article as: $\mathbf{B r} \mathbf{J}$ Gen Pract 2015; DOI: 10.3399/bjgp15X684445 


\section{How this fits in}

General practice is becoming increasingly complex yet there has not been any change in the length or nature of GP training, which remains the shortest of all the medical and surgical specialty training programmes. There is a need to develop a flexible workforce that trains and works across traditional primary and secondary care boundaries. Knowledge about how well training prepares GPS for their role could help inform plans for extended training. Extended training could ensure all trainees experience a wider variety of primary care settings as well as increased opportunity for involvement in the business aspects of practice including commissioning and leadership roles, improving preparedness of GPs. A mix of placements could help GPs get such variety but this must still allow for continuity with patients and the opportunity to establish strong team relationships, both of which are valued by GPs and important for building generalist skills. Such changes could help GP trainees to develop the specialist as well as generalist skills expected of the future workforce.

success with this approach with doctors, both in terms of increasing participation rates and achieving high-quality data. The sample was drawn from 350 GPs who had qualified with Severn Deanery between 2007 and 2010 , for whom records were available. GP trainees at the deanery are comparable to trainees nationally in terms of sex mix but achieve higher than average recruitment scores and Membership of the Royal College of General Practitioners pass rate. Initial sampling was purposive, seeking a mix of sex, level of experience, and geographical areas, with later selection being guided by data analysis. ${ }^{9}$

\section{Data collection and analysis}

Eighteen interviews were completed: four with partners, eight with salaried GPs, and six with locum GPs. Twelve GPs were female. This mix of roles and sex is typical of newly-qualified GPs in Severn and reflects the national feminisation of the GP workforce. All participants opted for a telephone interview, which lasted on average 46 minutes giving a rich data set. The topic guide was developed by the authors and reviewed by a service user involved in the wider project, to enhance the quality and relevance of the research. ${ }^{10}$ An outline of the topic guide is given in Appendix 1.

A thematic framework analysis of the data was undertaken, ${ }^{11}$ supported by the use of
NVivo (version 9). An initial coding framework was built up from the questions used as well as issues arising in discussion, and this was applied to the first eight interviews. To enhance rigour, the authors examined three transcripts independently and identified overlapping themes, helping to confirm the initial framework. The service user, who had experience of coding, also reviewed a sample of transcripts, showing strong consistency in the interpretation of transcripts and the use of codes. The framework was applied to subsequent data allowing for further refinements, consistent with this approach to data analysis. Disconfirming evidence was sought throughout the analysis. ${ }^{12}$ In the coding of the final four transcripts no adjustments were made to the framework, leading to closure of data collection. ${ }^{?}$

\section{RESULTS}

\section{Theme 1: The value of variety}

Working in different practices. Newlyqualified GPs place enormous value on learning the role of a GP through doing the job, both as a trainee and then in the early years of practice, during which they begin to develop their generalist skills. This was a distinct theme in all interviews and these two GPs capture the strength of feeling:

'There is nothing that can quite prepare you for how it is, you just have to do it I think.' (P4, partner)

I think there are certain things you simply have to do. You can't learn to drive by learning the Highway Code.' (P13, salaried)

However, what adds real value to experiences in practice is variety. Newlyqualified GPs gain enormously from experiencing different practices, as a registrar and, once qualified, through locum or other short-term positions. Variety helps trainees to not only see how other practices are run, but also the differences arising from geographical setting and social environment:

Working in the rural areas is really different because geography makes such a massive impact on your decisions whether to admit someone or not is suddenly not just clinical, it's geography.' (P2, locum/mixed role)

It was a very deprived area and so the type of things that you see are different.' (P27, salaried)

Most had not experienced such variety until after qualifying, so having only worked in one type of practice as a registrar they 
were surprised by the contrasts and benefits:

I thought I loved my registrar practice, I thought it was brilliant ... but I am so glad that I have experienced other practices now.' (P12, salaried)

'I think what's amazing is how different, different practices are really in terms of the team feel, the patient population ... I think it is really helpful just to see how different practices do things. '(P7, salaried)

Broadening experience in the GP role is clearly beneficial and in both clinical and non-clinical aspects helps newly-qualified GPs ease into the job:

I am more confident because of working in some different places and having to deal with a little more ... I feel better for it.' (P8, salaried)

Working in other primary care settings. Those who had experience of primary care roles outside GP practices also discovered how useful this could be. Roles mentioned included working in an acute GP support service based in secondary care triaging potential GP admissions, a referral management service, and prison work. Although this may be uncommon within GP training at present, some exposure to such roles can clearly be valuable for understanding the wider context of the role:

What was massively useful was doing ... the acute GP service in which I try to keep patients at home using lots of community services. Now when I started this job I didn't know anything about it and now / know a lot more and that's really useful for my ... other job in which I am a locum GP.' (P2, locum/ mixed role)

'It gives you a different perspective on different roles and expectations and demands of being a GP.' (P28, locum/mixed role)

In addition to learning about the wider role of the GP and broadening perspective of the NHS, adaptability was felt to be valuable:

'It is useful to perhaps move around a bit more ... you then learn how to adapt to that and learn what it is you need to know.' (P20, partner)

Theme 2: Importance of continuity

While newly-qualified GPs can clearly gain from greater variety in GP training, continuity with patients is hugely important and remained a strong theme. For most, experiencing continuity with patients was a vital element of working in general practice, and also brings enjoyment and rewards:

'The continuity of care is what I really like.' (P4, partner)

I would like to have some continuity with patients ... I don't like going in, seeing a patient the one time, never being able to follow them up and check or that sort of thing.' (P14, locum)

\section{Theme 3: Opportunities for management and leadership}

There was a definite readiness among these newly-qualified GPs for taking on management and leadership roles, and this grows with experience in the job:

As time goes on you feel able to devote some time to the other aspect, you know, the sort of business side of things and reducing referrals and thinking about how we plan services and things like that.' (P1. partner)

However, a key theme emerged around opportunity and how this can enhance or hinder the value of experiences in GP practice. As registrars and newly-qualified GPs, opportunities to be involved in the business of managing a practice vary. Some practices enable involvement and have a more inclusive approach, compared with others where opportunities are limited. Where opportunity is provided, trainees benefit from the experience:

When I was a trainee I did used to go to some of the business meetings in the practice as well as the clinical meetings and that was quite useful, even if I didn't always understand exactly what was going on, to kind of get a feel for how QOF affects the partners or how they make decisions about hiring new practice managers. (P1, partner)

This GP's experience is clearly in contrast to the one described below, consistent with others in this study:

'I was never invited to any partners meetings so I ... had little handle on what they actually discuss, the way they resolve things, what were the issues that they faced?' (P28 locum/mixed role) 
There is evidently a lack of consistency in whether GP registrars are given opportunities to observe or participate in the business or partner meetings, meaning that some will enter their first posts with little or no understanding of managing a general practice, and this is a pattern that can continue once qualified. A lack of opportunity in leadership roles can be similarly disempowering, as for this GP who talked of not yet being involved in the Quality and Outcomes Framework (QOF):

'That scares me a little about being a partner.' (P26, salaried)

Many seek to build relationships at work as a way to strengthen support with these additional parts of the GP role, developing skills around teamwork and delegation:

I think I do OK because perhaps I have a good enough relationship with the people that I'm asking to help do the work ... that makes it easier.' (P21, salaried)

We've got a pretty good relationship with our nurses so they will help me out when they can.' (P13, salaried)

As one newly-qualified GP suggested, a longer period of training could be helpful for giving more time for this type of work:

'Then in that last year of training you could take on more responsibility and you could take on more of a management role and a responsibility for QOF or something ... I don't think a year as a registrar can prepare you at all for being a partner. '(P12, salaried)

\section{Theme 4: Additional areas for GP specialty training}

As implied in the previous theme, the data revealed some gaps in training relating to the running of a practice and being a partner. More insight into business skills', 'how a practice runs', 'how to run a business', and how partnerships work, were all suggested as ways to enhance training:

If you were going straight into a partnership that's a huge... knowledge gap that we don't really have any experience in managing ... leading a team.' (P7, salaried)

Some people [fellow trainees] just had no idea that you even had to buy into a partnership.' (P12, salaried)

The financial side of business and how practices actually gain their income seems a particular mystery:

[At practice meetings] if it's purely money I really feel out of my depth, I've got no idea about the finances of the practice.' (P16, salaried)

These newly-qualified GPs also anticipated that, in future, GP training would benefit from addressing commissioning and this could fit as part of training around finances and the structure of the NHS. Those who had experience of this kind found it very helpful:

What this has done is really made me understand how the healthcare system works, how primary care works, how the changes are happening ... you feel slightly more empowered which is really nice.' (P2, locum/mixed rolel

\section{DISCUSSION}

\section{Summary}

The value of experience in primary care settings as part of the training for general practice is firmly endorsed by this study. Collectively, the data support the grounds for extending GP training, ${ }^{7}$ to give more time for becoming prepared for practice, and, furthermore, suggest ways in which additional time could enrich the training experiences of GPs such as increasing the variety of training settings and widening management opportunities. These findings may also be helpful more widely for those planning ways to broaden training experiences for all doctors who need to be prepared to work across the boundary of primary and secondary care, to work in multidisciplinary teams and develop generalist, transferable competencies. ${ }^{6.13}$

\section{Strengths and limitations}

The study was set in one location and it is possible that GPs who trained in other areas have different views. However, the sample included newly-qualified GPs working in a diverse range of communities, including inner city with areas of high deprivation, more affluent urban and suburban areas, and rural areas with more mixed populations. The rich account of views obtained from this sample, the achievement of saturation, and the consistency in the themes that emerged, further increases the confidence in transferability of these findings to newlyqualified GPs nationally.

\section{Comparison with existing literature}

The GPs in this study benefited from 
working in a variety of primary care settings both in and outside practices, helping them develop adaptability and boosting confidence and skills. However, not all trainees experience variety and it could be beneficial to ensure greater variety in primary care training experiences, both in and outside practices, for all trainees. Rughani et a ${ }^{14}$ offer further support for this and highlight that a longer period of training would facilitate opportunities. Experience of working in different practices does not need to be extensive; a few months in other locations can be beneficial in seeing how different practices are run and can have other advantages such as insight into different geographical locations, populations, and environments. In this way, training would broaden the perspective of diverse populations and the social context of health, fitting with the ethos of medical generalism in helping to deliver whole person medicine. ${ }^{15}$

For those who have opportunities to work in primary care outside GP practices, there can be additional gains in terms of understanding the wider NHS, its infrastructure, and financing. Such experience could further help prepare doctors to work collaboratively across community and acute care settings. ${ }^{6}$ Alongside the benefits of variety, it is important to note the finding that continuity of patients remains important to GPs, consistent with other research ${ }^{16,17}$ and also central to the concept of medical generalism, ${ }^{15}$ which makes it essential that variety and continuity are appropriately balanced in training experiences. Although these may seem incompatible, agreed minimum placement lengths (such as a few months) across the various placements offered could achieve this balance, fitting with the expectations of GP trainees who cite continuity with patients in their top three reasons for choosing a career in general practice. ${ }^{18}$

The importance of ensuring that all trainee GPs are given opportunities for management and leadership roles is highlighted by this study and fits with the call for management placements during specialist training for all doctors, ${ }^{6}$ and for leadership training for health professionals more generally. ${ }^{13}$ Experience of these roles during training varies widely and appears to be dependent on the management style and culture at the training practice. This means that some GPs enter their first posts in qualified practice with little or no understanding of managing a practice, the financing of health care, leading a team, or quality improvement, potentially delaying involvement in these areas and making it harder to develop the skills necessary to take on a leadership role inside the practice or elsewhere. Furthermore, in time, the repercussions of a divide between those who are given such opportunities and those who are not, may be seen in a deepening of a 'two tier' culture in general practice glimpsed in this study and elsewhere. ${ }^{19}$ Seeking good relationships and a team approach gives trainees support as well as honing teamworking skills. Griffin et a ${ }^{20}$ have similarly highlighted the importance of 'meaningful long-term clinical relationships' for new GPs. Efforts to strengthen relationships between new and experienced members of the team, and fully involve trainees in the wider primary care team and with outside organisations, will help them participate in team-based integrated care. This, together with widening participation in business and leadership activities, could improve the experience, skills, and knowledge of trainees, fitting with the aspirations of extended training and for a workforce with greater generalist skills. ${ }^{7.13}$

\section{Implications for practice}

Many of the themes in the results section highlight the considerable potential of extended training. First, this could incorporate greater variety in training experiences, widening insight into different patient populations and their geographical and social environments, and how these factors shape the work of a GP. A mix of placements in contrasting practices could achieve this, preparing trainees to care for a complex and diverse range of patients and deliver holistic care. Placements in primary care outside practices could also help broaden experience of care provision in other settings, as well as understanding of NHS infrastructure, financing, management, and planning services, filling some of the perceived gaps in training and facilitating cross-sector working and collaboration. GPs can expect to work across a variety of settings and broader experience will help them prepare for this and develop a flexible approach to work. However, it is important to be mindful that patient continuity emerged as a strong theme here, consistent with other research showing this as a 'core value' of GP work ${ }^{17}$ and essential to those considering a GP career. ${ }^{18}$ Therefore this should feature in planning extended training, with agreed minimum placement lengths.

Second, extended training could give 


\section{Box 1. Recommendations for planning extended training}

- Provide GP placements of a few months in different types of practice during the period of training; in addition, provide opportunities to work some sessions in other settings outside GP practice.

- Trainees to have a mentor from within the practice and/or outside, for example, clinical commissioning group/locality group, to support involvement in leadership activities, including a mandatory project in quality improvement or service development, as a requirement for Certificate of Completion of Training.

- Provide shadowing opportunities for all trainees to work with both clinical and non-clinical professionals involved in business aspects of practice management.

- Support practices with widening opportunities for newly-qualified sessional doctors to further develop their generalist and enhanced skills, and to be mentored by an experienced GP to help avoid a divide deepening between sessional doctors and partners. A toolkit has been developed for practices by Severn School of Primary Care, to support the personal and professional development of newlyqualified GPs, which could help to address this. ${ }^{21}$

- Develop opportunities for integration of training across different specialties as part of a broad-based training. For example, GP specialty trainees and specialist medical trainees working in community clinics, such as, psychiatry, paediatrics, and care of older people.

\section{Funding}

The larger study of which this was a part was jointly funded by Severn Postgraduate Medical Education, Health Education South West, and the University of the West of England, Bristol (HLS-NM-10-071).

\section{Ethical approval}

The study was approved by the UWE Faculty Research Ethics Sub-Committee in July 2011 (HSC/11/7/68). All participants gave their consent to take part in the interview and were assured of confidentiality.

\section{Provenance}

Freely submitted; externally peer reviewed.

\section{Competing interests}

The authors have declared no competing interests.

\section{Acknowledgements}

The authors wish to thank the service user for her interest and input, and all participants who generously gave their time to be interviewed. They are also grateful to the peer reviewers who commented on an earlier draft of this article.

\section{Discuss this article}

Contribute and read comments about this article: bjgp.org/letters careers. Making a leadership project, such as quality improvement, a recommended or even mandatory part of GP training, could be a way to ensure all trainees are engaged in such opportunities and develop an approach to lifelong learning.

A number of specific suggestions are offered that may help those responsible for planning extended training to realise these potential benefits (Box 1). The findings may also apply to the development of both clinical and non-clinical continuing training modules, and further research could usefully extend into innovative approaches to developing a GP career. training improves preparedness of the workforce as well as enhances individual varied opportunities in management leadership, practices could be supported in ways to involve and mentor trainees in specific roles and activities so that longer

greater opportunities for GPs to learn the full for the wide-ranging demands of the GP role as well as supporting the developm of generalist, transferable competencies, seen to be essential for tomorrow's
doctors.6,7,13 Given the findings about resce scope of the role and develop business and
leadership skills before the end of training. leadership skills before the end of training, varied opportunities in management and (1)

f




\section{REFERENCES}

1. Department of Health. High quality care for all: NHS next stage review final report. London: The Stationery Office, 2008.

2. Department of Health. NHS next stage review: our vision for primary and community care. London: DoH, 2008.

3. Department of Health. Transforming community services: enabling new patterns of provision. London: DoH, 2009

4. Royal College of General Practitioners. Curriculum for specialty training for general practice. The core statement: being a general practitioner. London: RCGP, 2007.

5. Department of Health. Modernising medical careers: the next steps. London: DoH, 2004.

6. Greenaway D. Securing the future of excellent patient care. Final report of the independent review. Shape of Training, 2013. http://wmw.shapeoftraining.co.uk/ static/documents/content/Shape_of_training_FINAL_Report.pdf_53977887.pdf laccessed 24 Jan 2015).

7. Royal College of General Practitioners. Preparing the future GP: the case for enhanced GP training. London: RCGP, 2012

8. Sabey A, Hardy H. Prepared for commissioning? A qualitative study into the views of recently qualified GPs. Educ Prim Care 2013; 24(5): 314-320.

9. Green J, Thorogood N. Qualitative methods for health research. London: Sage, 2004.

10. Staniszewska S, Brett J, Mockford C, Barber R. The GRIPP checklist: strengthening the quality of patient and public involvement reporting in research. Int J Technol Assess Health Care 2011; 27(4): 391-399.

11. Ritchie J, Spencer $L, O^{\prime}$ Connor W. Carrying out qualitative analysis. In Ritchie J, Lewis J, eds. Qualitative research practice: a guide for social science students and researchers. London: Sage, 2003: 219-262.

12. Huberman AM, Miles MB. The qualitative researcher's companion. Thousand
Oaks, CA: Sage, 2002.

13. Department of Health. Delivering high quality, effective, compassionate care: developing the right people with the right skills and the right values. A mandate from the Government to Health Education England: April 2014 to March 2015. https://uww.gov.uk/government/uploads/system/uploads/attachment_data/ file/203332/29257 2900971 Delivering Accessible.pdf laccessed 15 Jan 2015).

14. Rughani A, Riley B, Rendel S. The future of GP specialty training: enhanced and extended. Br J Gen Pract 2012; 62(599): 288-289.

15. Royal College of General Practitioners. Medical generalism: why expertise in whole person medicine matters. London: RCGP, 2012.

16. Ridd M, Shaw A, Salisbury C. 'Two sides of the coin' - the value of personal continuity to GPs: a qualitative interview study. Fam Pract 2006; 23(4): 461-468.

17. Stokes T, Tarrant C, Mainous AG 3rd, et al. Continuity of care: is the personal doctor still important? A survey of general practitioners and family physicians in England and Wales, the United States, and the Netherlands. Ann Fam Med 2005; 3(4): 353-359.

18. Irish B, Lake J. When and why do doctors decide to become general practitioners? Implications for recruitment into UK general practice specialty training. Educ Prim Care 2011; 22(1): 20-24

19. Lester $\mathrm{H}$, Campbell SM, McDonald R. The present state and future direction of primary care: a qualitative study of GPs' views. Br J Gen Pract 2009; DOI: 10.3399/bjgp09X473060

20. Griffin A, Abouharb T, Etherington C, Bandura I. Transition to independent practice: a national enquiry into the educational support for newly-qualified GPs. Educ Prim Care 2010; 21(5): 299-307.

21. Schroeder $\mathrm{K}$, Hardy H, Holmes S, et al. Surviving as a new GP: how to get the most out of your first years in practice. London: Royal College of General Practitioners, in association with NHS Health Education South West, 2013. http:// uww.rcgp.org.uk/rcgp-near-you/faculties/sw-england-and-wales-region/ / media/Files/RCGP-Faculties/SW-England-Wales/Severn/Surviving\%20as $\% 20$ \%20New\%20GP.ashx laccessed 17 Feb 2015). 


\section{Appendix 1. Topic guide for interviews}

- Expectations versus reality of practice (skills, qualities, and knowledge).

- Overview of experience - practices/settings they have worked in, adapting to new settings, and preferences; views about variety.

- Views, experiences, and involvement in management roles/tasks (meetings, team/wider relationships/ communication, IT); feelings about this part of the role.

- Views, experiences, and involvement in leadership tasks/activities; feelings about this part of the role.

- Personal/career issues - working in a team, confidence, support, workload, career planning, and preferences. 